\title{
Jaccard Coefficients based Clustering of XML Web Messages for Network Traffic Aggregation
}

\author{
Dhiah Al-Shammary \\ College of Computer Science and Information Technology \\ University of Al-Qadisiyah \\ Iraq. \\ d.alshammary@qu.edu.iq
}

Recived : $1 \backslash 4 \backslash 2019$

Revised : 18\4\2019

Accepted : 22\4\2019

Available online : $6 / 5 / 2019$

\begin{abstract}
This paper provides static efficient clustering model based simple Jaccard coefficients that supports XML messages aggregator in order to potentially reduce network traffic. The proposed model works by grouping only highly similar messages with the aim to provide messages with high redundancy for web aggregators. Web messages aggregation has become a significant solution to overcome network bottlenecks and congestions by efficiently reducing network volume by aggregating messages together removing their redundant information. The proposed model performance is compared to both K-Means and Principle Component Analysis (PCA) combined with K-Means. Jaccard based clustering model has shown potential performance as it only consumes around \%32 and \%25 processing time in comparison with K-Means and PCA combined with K-Means respectively. Quality measure (Aggregator Compression Ratio) has overcome both benchmark models.
\end{abstract}

Keywords: Jaccard Coefficients, XML, Clustering, Similarity Measurements 


\section{Introduction}

Web communication protocol is a significantly main medium to transfer information over the internet represented in XML messages [4, 8]. Recently, different kind of XML based protocols have been developed adding potential services to the network like video conference and surveillance in addition to secured and private peer to peer communication $[14,1,5]$. However, these protocols still require potential improvements and contributions to solve having the situation of network bottlenecks and congestions.

\subsection{Problem and Motivation}

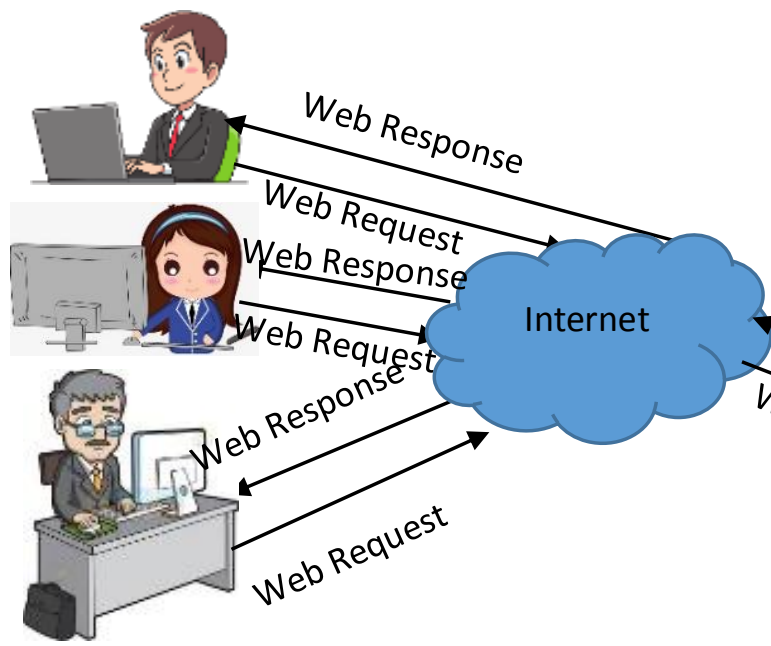

Figure 1: Jaccard Clustering Support for Stock Quote Web Servic

XML based Web messages representation has degraded the network performance by their highly redundant information creating high network traffic $[1,5,7,16]$. As a result of XML significant network volume, users have suffered from usual network bottlenecks and congestions [7, 2]. In many cases, network response time is very slow delaying user's services [5, 7, 8, 9]. This fact has motivated researchers and industrial engineers to adopt potential techniques that trying to reduce network traffic and speed up services response time. Web messages aggregator is one of the most recent proposed contributions attempting to catch group wise messages redundant information and creating one compact structure for a number of messages [10, 4]. However, potential aggregation depends on the similarity level of grouped messages. Therefore, a similarity measurement model is required to guarantee high aggregation results. An efficient clustering would support this requirement and improve Web services to endpoints over the network.

\subsection{Contribution}

This paper proposes an efficient clustering model for XML messages based on Jaccard coefficients similarity measurement. This similarity measurement has very low complexity that can guarantee a high network response time. Technically, Jaccard coefficients is calculated for two messages only. However, the proposed model has utilized this similarity metric to find the similarity of more than two messages and group them together. As a result, Web aggregator do not need to worry about finding similar messages and would only aggregate highly similar messages that can produce efficient network traffic reduction. Figure 1 illustrates a suggested scenario for 


\subsection{Organization of the Paper}

The rest of this paper is organized into five sections. Section 2 illustrates the related work and how other researchers attempted to cluster $\mathrm{Web}$ messages. Section 3 explains in details the proposed Jaccard clustering model. Section 4 presents a deep analysis and evaluation results of the proposed model. Finally, section 5 summarizes a conclusion of overall the paper.

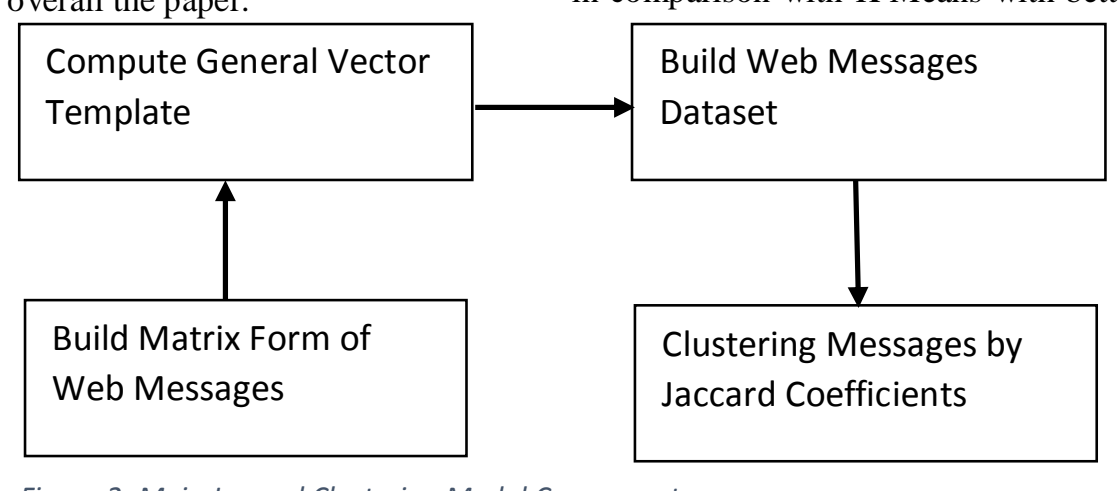

Figure 2: Main Jaccard Clustering Model Components

\section{Related Work}

Several studies have been achieved to provide potential clustering that can group numbers, messages or documents together based on how close they are to each other. A main direction of the developed clustering models is dedicated to cluster XML messages and documents. At the same time, another main direction of clustering techniques is dedicated to work on numbers and cannot work on text unless these textual documents are transformed to a related binary form.

$\mathrm{K}-\mathrm{Means}$ is one of the best known model based on Euclidian distance function. K-Means selects messages to be centroids and then search the closest messages to be linked with centroid message by having the shortest Euclidian distance. Another study is achieved to enhance K-Means by introducing Principle Component Analysis as a pre-processing to $\mathrm{K}$-Means. PCA is efficient in

$$
\begin{aligned}
& \text { < StockQuoteResponse > } \\
& \text { < StockQuote > } \\
& \text { < Company > AFI } \\
& \text { </Company > } \\
& \text { < Quotelnfo > } \\
& \text { < Price > 20.06 </Price > } \\
& \text { < LastUpdated > 01/09/2010 } \\
& \text { </LastUpdated > } \\
& \text { </Quotelnfo > } \\
& \text { </StockQuote > } \\
& \text { </StockQuoteResponse > }
\end{aligned}
$$

summarizing data bringing the main information into beginning of its results placement. Data with two-dimensional numbers, PCA will transform them into the same size two-dimensional numbers with $\% 80$ of the main information summarized in the first two columns. This fact has been utilized to summarize data and cluster them using only the first \%80 information located in the first and second columns. This has created more complexity in comparison with K-Means with better clustering results

XML messages have gained high interest of researchers and industrial engineers to cluster them directly working on the XML structure and format. Yongming [8] has developed a novel XML based clustering model. They have utilized vector space model with some extensions to capture the similarity parts of XML messages. Their proposed model works on a transformed XML dataset created by extracting features like recording leaf nodes path and weight. The empirical results have shown enhancements to the clustering job. Another model is presented by Hwang and $\mathrm{Gu}$ [10] by catching large XML structures and find out their frequencies inside the XML tree and other related tress. Their model works on other traditional features like XML nodes path. They cluster XML messages based on the higher similar frequencies of large XML structures in addition to tags and items. Their results have proven that they enhanced the clustering requirement and provide better results.

\section{Proposed Model}

The proposed technique consists of two main concepts: preparing XML messages into binary representation based on a generic numeric template and clustering messages based on Jaccard similarities using a predefined cluster size. Figure 2 shows the main processing components. 


\subsection{Dataset Transform}

Datasets usually have a specific format that is required for a specific clustering model. In this research, the first step is building XML trees of the considered XML messages. Figures 3 and 4 represent a real XML message example and its XML tree representation respectively. Then, XML matrix form is generated by level order traversing XML messages. Figure 5 shows the XML matrix form of XML tree. XML time series representation would be calculated using their XML matrix forms.

Distinctive items of all XML messages are collected to build their generic vector template in preparation to build the binary dataset of XML messages. Equation 1 illustrates the general shape of the generic vector template.

$$
\begin{aligned}
& T \\
& =\left\{\text { item }_{1}, \text { item }_{2}, \text { item }_{3}, \ldots, \text { item }_{n}\right\}
\end{aligned}
$$

Equation 2 illustrates the overall representation of dataset based on the weighted XML items (frequencies).

$$
\begin{aligned}
& X_{1}=\left\{w_{1}, w_{2}, w_{3}, \ldots, w_{n}\right\} \\
& X_{2}=\left\{w_{1}, w_{2}, w_{3}, \ldots, w_{n}\right\} \\
& X_{3}=\left\{w_{1}, w_{2}, w_{3}, \ldots, w_{n}\right\} \\
& \cdot \\
& \cdot \\
& X_{m}=\left\{w_{1}, w_{2}, w_{3}, \ldots, w_{n}\right\}
\end{aligned}
$$

The Term Frequency with Inverse Document Frequency (TF-IDF) is applied to calculate wi as illustrated in Eq. 3.

$$
w_{i}(d)=t f_{i} \times \log \frac{D}{d f_{i}}
$$

Where

- $\mathrm{tf}_{\mathrm{i}}$ is the frequency of XML item inside the document $d$.
- $\mathrm{df}_{\mathrm{i}}$ is the frequency of documents having instances of XML item.

- $\quad \mathrm{D}$ is the total number of involved documents in the whole dataset

\subsection{Jaccard Coefficients Grouping}

Jaccard Coefficients also known as Jaccard Index is a statistical factor that is used to find out similarity of two sets. It is widely applied to work on two sets only (Web messages). In this paper, Jaccard coefficient is applied as a base similarity function to work on a group of messages. The proposed clustering strategy is applied by selecting one of the involved XML messages as a centroid for each targeted cluster. Then, based on a pre-defined cluster size, the proposed model allocates messages based on their Jaccard similarity with the centroid message. Equation 4 illustrates the Jaccard Coefficient similarity metric of two sets $\mathrm{A}$ and $\mathrm{B}$.

$$
J(A, B)=\frac{|A \cap B|}{|A \cup B|}
$$

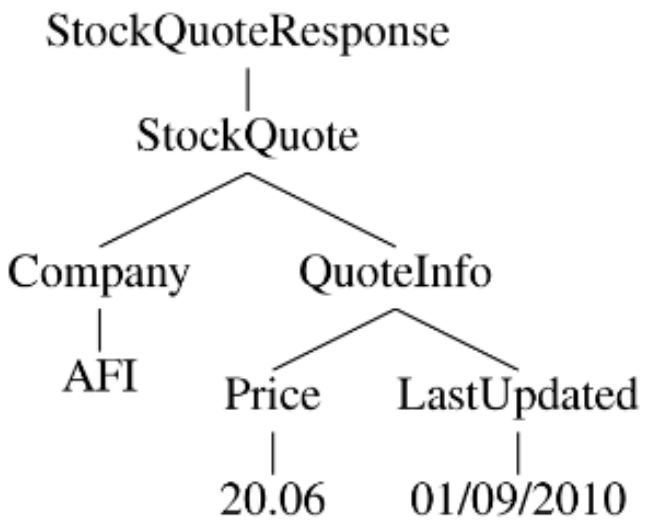

Figure 4: XML Web Message Tree

XML messages first transformed to generate dataset numerical representation. Equation (4) can be simplified into Eq. 5 .

$$
J(A, B)=\frac{|A \cap B|}{|A|+|B|-|A \cap B|}
$$


Dhiah .A

\begin{tabular}{|c|c|c|}
\hline Index & Node Content & $\begin{array}{c}\text { Parent } \\
\text { Index }\end{array}$ \\
\hline 0 & StockQuoteResponse & 0 \\
\hline 1 & StockQuote & 0 \\
\hline 2 & Company & 1 \\
\hline 3 & Quotelnfo & 1 \\
\hline 4 & AFI & 2 \\
\hline 5 & Price & 3 \\
\hline 6 & LastUpdated & 3 \\
\hline 7 & 20.06 & 5 \\
\hline 8 & $01 / 09 / 2010$ & 6 \\
\hline
\end{tabular}

Figure 5: Matrix Form of XML Web Message

The proposed model clusters messages based on their generated numerical representation. First, randomly selected messages are distributed into clusters as centres. Then Jaccard coefficients are calculated to the rest of messages in relation to centre points. Finally, messages are allocated to clusters based on the most similar Jaccard metric against the centre message.

\section{Experiments and Discussions}

The experiments to testify the proposed Jaccard coefficients clustering has used XML dataset published by [2] that consist of four groups 
Dhiah .A

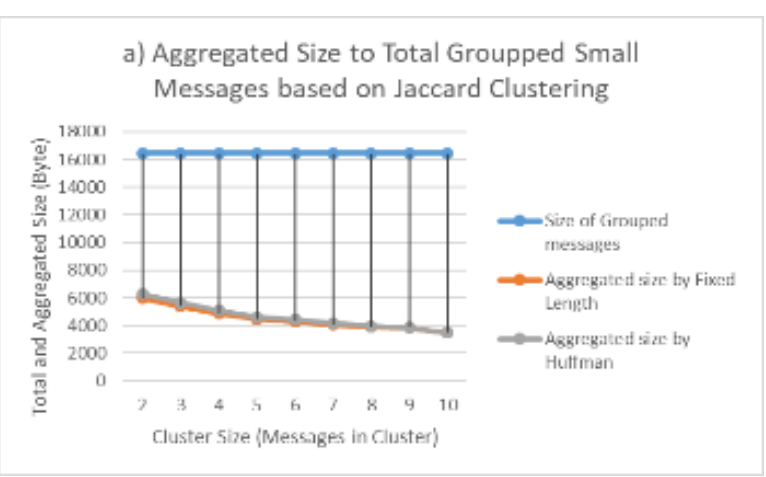

c) Aggregated Size to Total Groupped Large Messages based on Jaccard Clustering

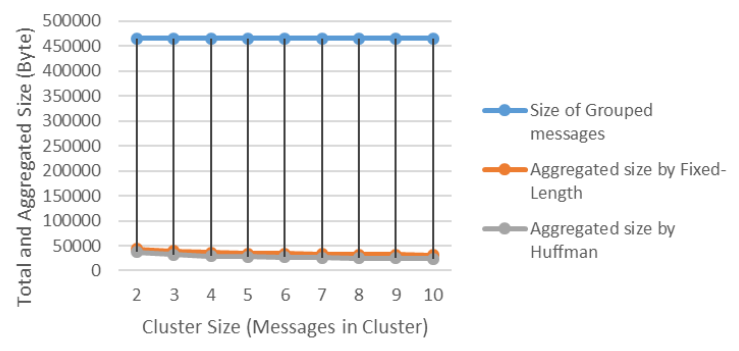

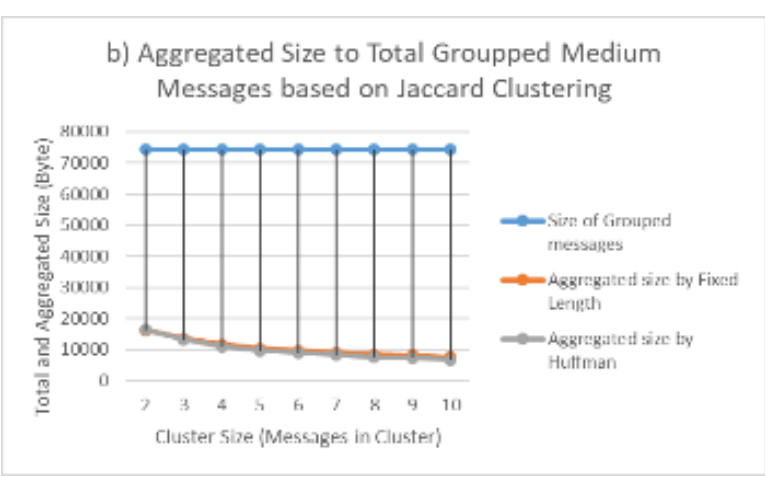

d) Aggregated Size to Total Groupped V.Large Messages based on Jaccard Clustering

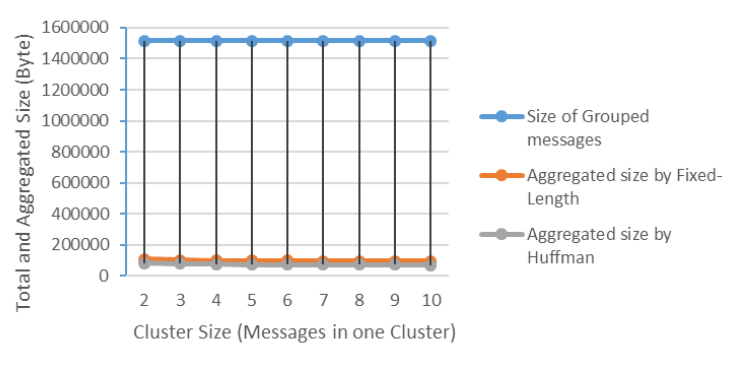

Figure 7: Aggregated Web messages sizes based on Jaccard Clustering

(small, medium, large, and very large) of messages. Messages have been distributed based on their size as potentially small messages like only 140 bytes allocated to small group and potentially large messages like $53 \mathrm{~KB}$ allocated to very large group. The evaluation strategy for the proposed model is set to test its resultant clusters by Web messages aggregator achieved by [16]. The same aggregator has applied to messages clusters generated by KMeans and PCA combined with K-Means clustering respectively. Aggregator resultant size reduction, compression ratios and clustering processing time are the main metrics to test our proposed model performance. Overall, the proposed model has potentially outperformed other models.

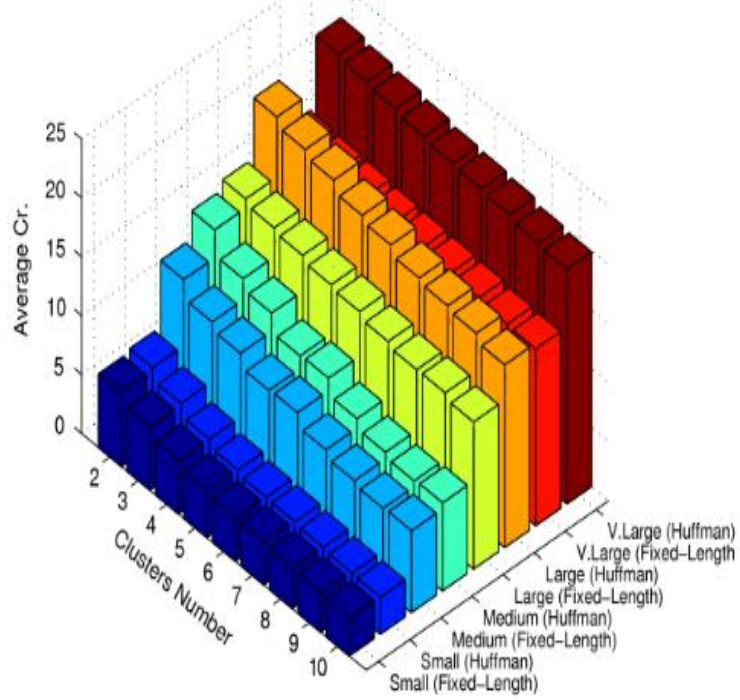

Figure 6: Aggregated Web messages based on K-Means and KMeans + PCA clusterina 


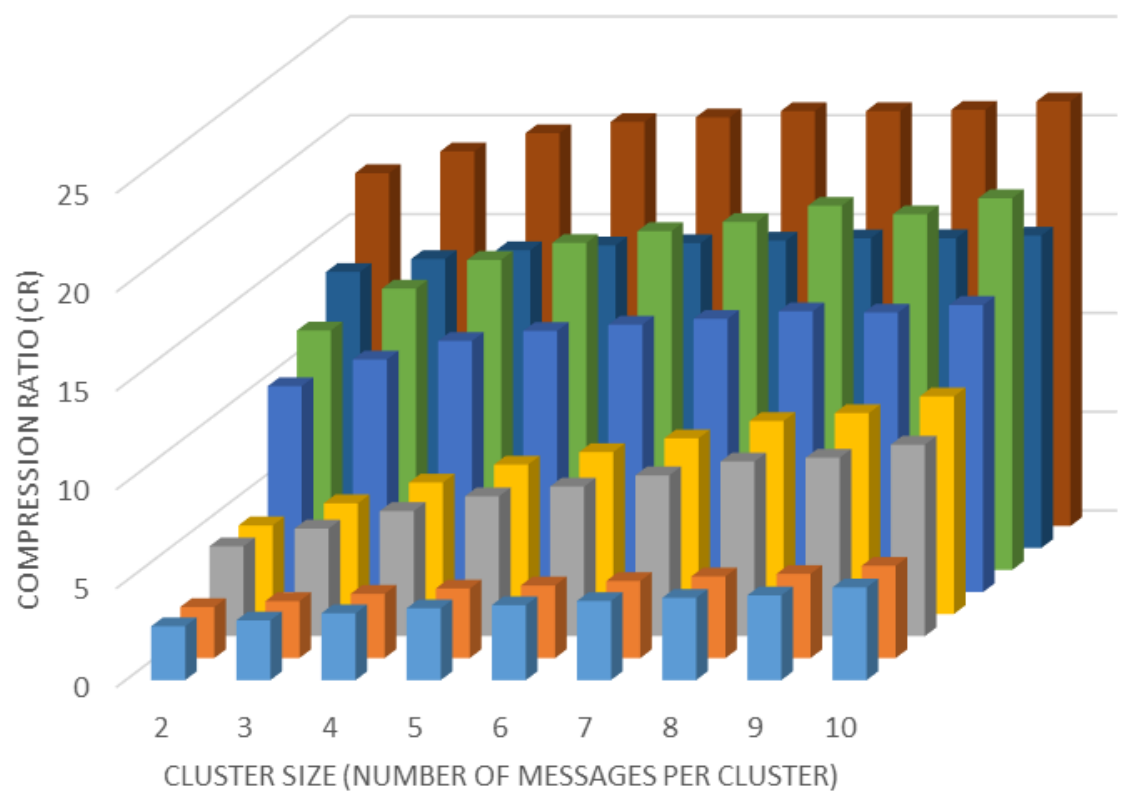

- Small Msg (Fixed-Length)

- Small Msg (Huffman)

Medium Msg (Fixed-Length)

- Medium Msg (Huffman)

- Large Msg (Fixed-Length)

- Large Msg (Huffman)

- V.Large (Fixed-Length)

- V.Large (Huffman)

Figure 8: Average Compression Ratios of Aggregated messages based on Jaccard Clustering

Figure 6 shows detailed observation for the Jaccard clustering size reduction in comparison with accumulated size of grouped messages without any size reduction. The results show a tremendous network size reduction that can significantly improve network performance and solve network bottlenecks and congestions. Table 1 provides a summary of the overall average compression ratio and clustering time for all models. Our proposed model has competitive compression ratios with K-Means and PCA combined K-Means as has generally outperformed them. Moreover, Jaccard clustering has potentially outperformed other benchmark models by processing time as it only requires about $\% 32$ and $\% 25$ of K-Means and PCA combined K-Means

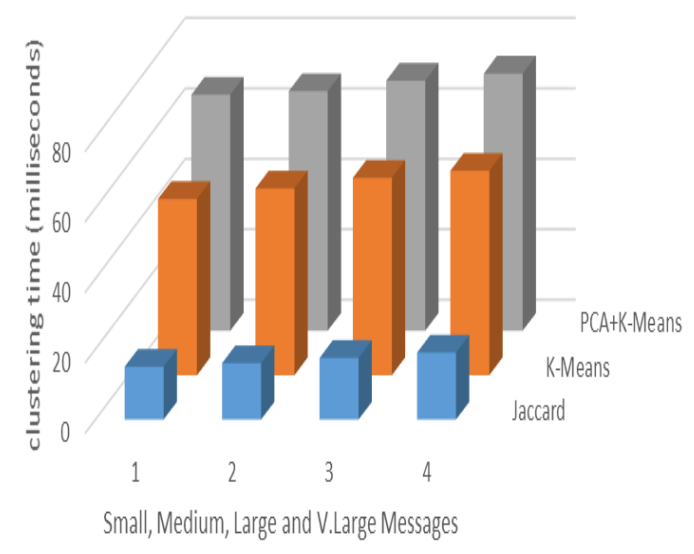

processing time respectively. Figures 7 and 8 shows detailed compression ratios for all the involved models and clearly Jaccard clustering has outperformed other techniques. Figure 9 illustrates the processing time in comparison with other benchmark models as the proposed technique has potentially outperformed them.

\section{Conclusions}

Web messages have been generated and used significantly over the network to exchange information. Generally, networks suffer from bottlenecks and congestions. Aggregation of Web messages would improve networks potentially. Efficient clustering would be a significant alternative to basic similarity measurements. This paper has proposed Jaccard Coefficient for clustering based on a predefined cluster size. Experiments have shown great achievement by the proposed model in comparison with both K-Means and PCA combined with K-Means as it outperformed them in both resultant compression ratios and clustering time. For future work, we are planning to deploy Jaccard clustering inside a large scale inter-cloud and measure its real-time performance. 
Table 1: Average compression ratio and clustering time of K-Means, PCA combined K-Means, and Jaccard based clustering

\begin{tabular}{|l|l|l|l|}
\hline $\begin{array}{l}\text { Cr and Time } \\
\text { Consumption }\end{array}$ & K-Means & $\begin{array}{l}\text { PCA+K- } \\
\text { Means }\end{array}$ & Jaccard \\
\hline Small Messages & \multicolumn{2}{l|}{} \\
\hline Cr (Fixed-Length) & 3.92 & 3.85 & 3.75 \\
\hline Cr (Huffman) & 3.82 & 3.71 & 3.65 \\
\hline Clustering Time (ms) & 50.88 & 65.33 & 15 \\
\hline Medium Messages & 6.77 & 6.8 & 7.4 \\
\hline Cr (Fixed-Length) & 7.72 & 7.84 & 8.04 \\
\hline Cr (Huffman) & 52.33 & 62.89 & 16 \\
\hline Clustering Time (ms) & \multicolumn{5}{l}{} \\
\hline Large Messages & 12.94 & 12.82 & 13.15 \\
\hline Cr (Fixed-Length) & 16.02 & 16.28 & 16.51 \\
\hline Cr (Huffman) & 54 & 68.11 & 17.5 \\
\hline Clustering Time (ms) & 15.12 & 15.13 & 15.23 \\
\hline V.Large Messages & 20.16 & 20.25 & 20.26 \\
\hline Cr (Fixed-Length) & 53.62 & 70.44 & 19 \\
\hline Cr (Huffman) & \multicolumn{5}{l}{} \\
\hline Clustering Time (ms) & \multicolumn{5}{l}{} \\
\hline
\end{tabular}

\section{References}

AK, Mishra J. Enhancing the beauty of fractals, ICCIMA'99. In: Proceedings. Third internatiфnjal conference, New Delhi, India, vol.16, issue 4, 2326 September 1999. p.454-8.

Al-Shammary D, KhalilI, George L. Clustering SOAP web services on internet computing usin\$8] fast fractals.In:201110 the IEEE international symposiumon network computing and applications (NCA),2011.p.366-71.

[3] C, Yang Z, Peng Z, Hua D, Xiaoxiao H, Zheng W, Junliang C. Development of web- telecom based hybrid services or chest ration and execution middleware over convergence networks.JNetworkComputApp12010;33:620-3010] C. Werner, C. Buschmann, Compressing SOAP messages by differential encoding, Web services, 2004, IEEE International Conference on Wab services, pages 540-547

D. Davis, M. Parashar, Latency performance soap implementations, in: $2^{\text {nd }}$ IEIEE/ACIN. International Symposium on Computing and the Grid, 2002, Dhiah Al-Shammary, Ibrahim Redundancy-aware SOAP compression and aggregation for enhance

performance, Journal of Network ar Computer Applications, Volume 35, Issue 2012, Pages 365-381

Dikaiakos M, Katsaros D, Mehra P, Pallis 1 Vakali A. Cloud computing :distributed intern computing for it and scientific research .IEE Internet Comput 2009;13:10-3.

G. Yongming, C Dehua, L. Jiajin, Clusterir XML Documents by Combining Content ar Structure, Volume 1, 2008, Pages 583-587 J.C. Hart, Fractal image compression ar recurrent iterated function systems, in: Computer Graphics and Applications, vc 16, IEEE Computer Society Press L Alamitos, CA, USA, 1996, pp. 25-33.

J.H. Hwang, M.S. Gu, Clustering $\mathrm{xml}$ documents based on the weight of frequent structures, Nov. 2007, pp. 845-849.

M.N. Jain, A.K. Murty, P.J. Flynn, Data clustering: a review, ACM Computing Survey 31 (3) (1999) 264-323.

Abu-Ghazaleh, M. Lewis, Differential Clustdeserialization for optimized soap performance, in: Supercomputing, 2005, in: Proceedings of the Khal ACM/IEEE SC 2005 Conference, Nov. 2005, p. 21 [ 1 håssag. S. Flesca, G. Manco, E. Masciari, L. Pontieri, A. 
Pugliese, Fast detection of xml structural similarity, IEEE Transactions on Knowledge and Data Engineering 17 (2) (2005) 160-175.

[14] V, Makris C, Panagis Y, Sakkopoulos E. Techniques to support web service selection and consumption with QoS characteristics. J Network Comput Appl 2008;31: 108-30.

[15] X. Cui, T. Potok, P. Palathingal, Document clustering using particle swarm optimization, Jun. 2005, pp. 185-191.

[16] Z.T. Khoi, Anh Phan, P. Bertok, Similarity-based soap multicast protocol to reduce bandwidth and latency in web services, IEEE Transactions on Services

Computing 1 (2) (2008) 88-103. 
Dhiah .A

تجميع وتقليل احمال الثبكات باستحداث مصنف رسائل الاكس ام ال باعتماد معامل التثابه لجاكارد

م.د ضياء عيدان جبر الشمري

قسم علوم الحاسوب / كلية علوم التحاسوب وتكنولوجيا ألمعلومات / جامعة القادسية

d.alshammary@qu.edu.iq

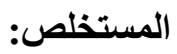

يقام هذا البحث مصنف جديد لاعم عمليات تجميع وتقليل احمال الثبكات الخاصة برسائل الاكس ام ال بالاعتماد على معامل تثلثابه جاكارد.

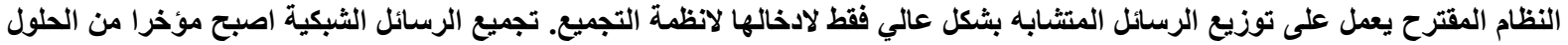

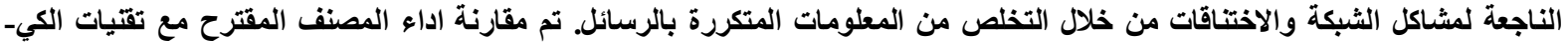

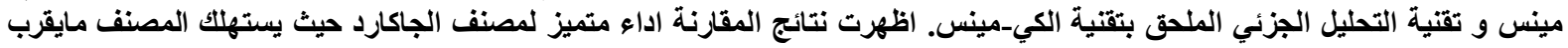
بمعدل 32\% و 25\% مما يستهلكه وقت المعالجة لتقنيات الكي-مينس وتقنية التحليل الجزئي الملحقة بتقية الكي-مينس بالتتابع. كذلك النتائج اظهرت نسبة ضغط تجاوزت ماانجز مع التقنيات المقارنة. 